\title{
AÇÕES E REPERCUSSÕES DAS PRIMEIRAS INICIATIVAS DAS UNIVERSIDADES PÚBLICAS FEDERAIS BRASILEIRAS FRENTE À COVID-19
}

\author{
P. T. C. DE O. SALVADOR ${ }^{1}$, K. Y. A. ALVES ${ }^{2}$, C. C. F. M. RODRIGUES 3 , L. V. E OLIVEIRA ${ }^{4}$, V. R. M.DE SOUSA ${ }^{5}$ \\ Universidade Federal do Rio Grande do Norte \\ ORCID ID: https://orcid.org/0000-0002-3208-6270 \\ petalatuani@hotmail.com ${ }^{1}$
}

Submetido 14/03/2020 - Aceito 26/08/2020

DOI: $10.15628 /$ holos.2020.10791

\section{RESUMO}

Objetiva-se mapear a publicização das primeiras ações realizadas pelas universidades públicas federais brasileiras frente à COVID-19 e suas repercussões nas redes sociais. Trata-se de pesquisa exploratóriadescritiva, de abordagem quanti-qualitativa, realizada em contexto web, no mês de abril de 2020, a partir de dados coletados nas redes sociais digitais das universidades públicas federais brasileiras. Os dados quantitativos foram analisados a partir de estatística descritiva simples e os qualitativos - comentários - a partir de lexicografia com suporte de software. Avaliouse 3.576 postagens relacionadas à COVID-19, das quais $2.215(61,9 \%)$ foram divulgadas no Facebook e 1.361 $(38,1 \%)$ no Instagram. Os dados revelaram um incremento na publicização de postagens sobre o COVID19 a partir do mês de março, com predominância das postagens do tipo "ações", como: suspensão de atividades acadêmicas/administrativas, trabalho remoto, apoio psicológico à sociedade, produção de máscaras protetoras e preparações alcoólicas, concessão de espaços para instalação de hospitais de campanha, atuação de Hospitais Universitários, entre outros. A análise lexicográfica dos comentários resultou em duas classes: "Angústias antes da suspensão das atividades e reconfigurações do funcionamento da universidade" e "Orgulho pela atuação da universidade". Elucidou-se que as universidades públicas federais brasileiras têm atuado ativamente em ações de enfrentamento à COVID-19, desde reorganizações acadêmicas e administrativas para contribuir com a minimização do risco a sua comunidade e região, até ações sociais de suporte à comunidade acadêmica, sobretudo estudantes, bem como pesquisas básicas e aplicadas relacionadas à temática, o que tem sido decisivo no contexto brasileiro.

PALAVRAS-CHAVE: Pandemias, Infecções por coronavírus, Pesquisa, Rede social, Universidades.

\section{ACTIONS AND REPERCUSSIONS OF THE FIRST INITIATIVES OF BRAZILIAN FEDERAL PUBLIC UNIVERSITIES AGAINST COVID-19}

\section{ABSTRACT}

The objective is to map the publication of the first actions carried out by Brazilian federal public universities against COVID-19 and its repercussions on social networks. This is an exploratory-descriptive research, with a quantitative-qualitative approach, carried out in a web context, in April 2020, based on data collected in the digital social networks of Brazilian federal public universities. The quantitative data were analyzed from simple descriptive statistics and qualitative statistics comments - from lexicography with software support. A total of 3,576 posts related to COVID-19 were evaluated, of which $2,215(61.9 \%)$ were posted on Facebook and 1,361 (38.1\%) on Instagram. The data revealed an increase in the publication of posts about COVID-19 from March, with predominance of posts of the type "actions", such as: suspension of academic/administrative activities, remote work, psychological support to society, production of protective masks and alcoholic preparations, granting spaces for the installation of campaign hospitals, performance of University Hospitals, among others. The lexicographic analysis of the comments resulted in two classes: "Anguish before the suspension of activities and reconfigurations of the functioning of the university" and "Pride for the performance of the university". It was learned that Brazilian federal public universities have actively acted in actions to combat COVID-19, from academic and administrative reorganizations to contribute to the minimization of risk to their community and region, to social actions to support the academic community, especially students, as well as basic and applied research related to the theme, which has been decisive in the Brazilian context.

KEYWORDS: Pandemics, Coronavirus Infections, Research, Social Networking, Universities. 


\section{INTRODUÇÃO}

A Organização Mundial de Saúde (OMS), em 11 de março de 2020, caracterizou como pandemia a doença causada pelo novo coronavírus SARS-CoV-2, denominada como COVID-19 (Gallasch, Cunha, Pereira, \& Silva-Júnior, 2020). Por possuir comportamento errático, alta taxa de transmissibilidade e taxa de letalidade estimada em $3,4 \%$, semelhante à da gripe espanhola e muito mais elevada do que a da influenza A H1N1 $(0,02 \%)$ e da gripe sazonal $(0,1 \%)$, a pandemia de COVID-19 configura-se em um desafio para saúde pública mundial (Silva, 2020; Freitas, Napirroga, \& Donalísio, 2020).

Frente ao conhecimento de que a transmissão da COVID-19 é favorecida pelo contato próximo e desprotegido com secreções ou excreções de pessoas infectadas, principalmente por meio de gotículas salivares; bem como à impossibilidade de controle imediato da pandemia por redução de susceptíveis por meio de vacinação; e à inexistência de medicamentos comprovadamente eficazes para tratar os doentes, as autoridades sanitárias mundiais têm sugerido a adoção de medidas de isolamento físico social, a exemplo do fechamento de comércios, escolas e universidades, a fim de diminuir a propagação da COVID-19 (Rafael et al., 2020).

No Brasil, o contexto de desigualdade social e demográfica, com populações vivendo em condições precárias de habitação e saneamento, associado à alta prevalência de doenças crônicas, potencializam os desafios para o enfrentamento desta pandemia, assim se torna mister o comprometimento da comunidade científica na mobilização de iniciativas que tanto possam subsidiar a tomada de decisão de agentes públicos envolvidos no controle da doença, quanto beneficiar a sociedade em geral (Barreto et al., 2020).

Nesse contexto, estão inseridas as universidades públicas federais, as quais possuem o compromisso social de mobilizar suas infraestruturas, funcionários de diferentes áreas, alunos e pesquisadores para condução de iniciativas que minimizem os efeitos negativos da doença (Gimenez, Sousa, \& Feltri, 2020).

Tais iniciativas, por sua vez, em consonância ao crescente uso de tecnologias da informação e da comunicação (TIC), são corriqueiramente divulgadas para além do cenário acadêmico, incluindo redes sociais, o que pode dinamizar o fluxo de dados e informações para a tomada de decisão, para a produção do conhecimento em redes e para ampliar os canais de comunicação e democratização do conhecimento científico (Barcelos, Lima, \& Aguiar, 2020).

Nesta perspectiva, as seguintes questões de pesquisa foram traçadas: Quais as ações estão sendo realizadas e divulgadas nas redes sociais das universidades públicas federais brasileiras frente à COVID-19? Qual tem sido a repercussão dessa publicização? Objetiva-se, portanto, mapear a publicização das primeiras ações realizadas pelas universidades públicas federais brasileiras frente à COVID-19 e suas repercussões nas redes sociais. 


\section{METODOLOGIA}

Trata-se de pesquisa exploratória-descritiva, de abordagem quanti-qualitativa, realizada em contexto web, método que compreende que, com a utilização da Internet, é possível estudar as relações nos espaços virtuais, como também explorar o cotidiano de grupos específicos compostos por características em comum (Feitosa \& Araújo, 2014).

Utilizou-se como fonte de coleta de dados as redes sociais digitais - Instagram e Facebook - das universidades públicas federais brasileiras. Redes sociais digitais são grupos de pessoas que trocam informações, ideias e conhecimento, em torno de um objetivo em comum, gerando novas maneiras de participação política, de entretenimento e de contato social, utilizando a mediação tecnológica na comunicação (Santos \& Santos, 2014).

Inicialmente, foi realizado levantamento das universidades públicas federais no Cadastro Nacional de Cursos e Instituições de Educação Superior (e-MEC), no dia 06 de abril 2020. Identificou-se a existência de 67 instituições. Para acessar o Instagram e Facebook das universidades, acessou-se o site oficial da instituição e, a partir dele, buscou-se os links de acesso das redes sociais oficiais da instituição. Das 67 universidades, seis não possuíam Instagram ativo, três não possuíam Facebook ativo e uma não possuía nem Instagram nem Facebook ativo no período da coleta de dados. Desse modo, foram analisadas as redes sociais de 66 universidades, sendo 61 perfis de Instagram e 64 de Facebook.

Após o acesso à rede social oficial da instituição, foi identificada a primeira postagem sobre a temática COVID-19 em cada rede social, com o objetivo de traçar a linha do tempo de quando a instituição começou a publicizar postagens sobre a temática. Após isso, foram analisadas todas as postagens a partir da primeira até o dia 06 de abril de 2020 - data em que o processo de coleta de dados foi iniciado. A coleta de dados aconteceu de 06 a 27 de abril de 2020.

Foram incluídas todas as postagens que abordavam a temática COVID-19 e seus comentários, tanto as que divulgavam notícias quanto as que publicizavam ações e recomendações. Excluíram-se as publicações de stories, os comentários em língua estrangeira e os comentários que não eram textuais (imagens, gifs, links etc.).

Cada uma das postagens selecionadas foi analisada de acordo com os indicadores de coleta de dados, a partir de planilha construída no Microsoft Excel 2020: universidade; rede social; data da postagem; tema da postagem (se ação, recomendação e/ou divulgação); quantidade de curtidas ou visualizações; quantidade de compartilhamentos; e quantidade de comentários (total). Quanto ao tipo de postagem, considerou-se: "ação" as que veiculavam proposições da própria universidade; "divulgação" se trazia ações de outras instituições; e "recomendação" se trouxesse orientações para prevenção.

Além disso, todos os comentários textuais disponíveis das postagens analisadas foram transcritos para documento do LibreOffice Writer, segundo formatação recomendada pelo tutorial para uso do software Interface de R pour les Analyses Multidimensionnelles de Textes et de Questionnaires (IRaMuTeQ) (Camargo \& Justo, 2018).

Os dados quantitativos foram analisados a partir de estatística descritiva simples e os comentários disponíveis foram analisados a partir de lexicografia com suporte do IRaMuTeQ. 
Utilizou-se o método de "parágrafos" para construção dos segmentos de texto e a Classificação Hierárquica Descendente (CHD) e a análise de similitude das classes como métodos de análise dos comentários. A CHD é um tipo de análise em que os segmentos de texto são classificados em função dos seus respectivos vocabulários e o conjunto deles é repartido em função da presença ou ausência das formas reduzidas, formando classes. Para a análise destas classes, utilizou-se a análise de similitude, que permite identificar as coocorrências entre as palavras e seu resultado traz indicações da conexidade entre as palavras (Camargo \& Justo, 2018).

Não foi necessária apreciação ética, por se analisar dados de domínio público.

\section{RESULTADOS E DISCUSSÕES}

No período analisado, as universidades públicas federais brasileiras publicizaram 3.576 postagens relacionadas à COVID-19, das quais 2.215 (61,9\%) foram divulgadas no Facebook e 1.361 $(38,1 \%)$ no Instagram. Quanto à distribuição destas postagens por região brasileira em que a universidade está inserida, as instituições da região Sudeste tiveram mais publicações (1.125; $34,3 \%)$, seguidas pelas do Nordeste $(924 ; 25,8 \%)$, Sul $(837 ; 23,4 \%)$, Norte $(348 ; 9,7 \%)$ e CentroOeste $(242 ; 6,8 \%)$.

Revelou-se um importante alcance destas postagens, as quais foram curtidas/visualizadas 2.755.687 vezes. Apesar de as universidades publicarem mais em seus perfis do Facebook, verificou-se maior engajamento dos usuários no Instagram, já que, desse total de curtidas/visualizações, 2.196 .875 (79,7\%) foram em postagens publicadas nesta rede social. Adicionalmente, porém, no Facebook é possível avaliar a quantidade de compartilhamento das postagens, que totalizou 224.395, aspecto que também contribui para o alcance das publicações das universidades.

No que se refere à repercussão destas postagens, evidenciou-se um total de 58.822 comentários, dos quais 31.662 (53,8\%) foram em publicações divulgadas no Facebook e 21.160 $(46,2 \%)$ no Instagram. A média de divulgação de postagens e de comentários por região brasileira em que as universidades estão localizadas pode ser visualizada na Tabela 1. Observa-se que as instituições das regiões Sudeste e Sul utilizam com expressividade o Facebook para publicização, enquanto no Nordeste é visível a repercussão do uso do Instagram pelas universidades desta região.

Tabela 1: Média de postagens e comentários, segundo rede social e região das universidades públicas federais brasileiras. Natal, RN, Brasil, 2020. $(n=66)$.

\begin{tabular}{|c|c|c|c|c|c|c|}
\hline \multirow[b]{2}{*}{ Variável } & \multicolumn{5}{|c|}{ Região ( $\bar{X} \pm D P)$} & \multirow{2}{*}{$\begin{array}{l}\text { Total } \\
(\bar{X} \pm D P)\end{array}$} \\
\hline & $\begin{array}{l}\text { Centro- } \\
\text { Oeste }\end{array}$ & Nordeste & Norte & Sudeste & Sul & \\
\hline \multicolumn{7}{|l|}{ Instagram } \\
\hline Postagem & $15,4 \pm 9,7$ & $26,2 \pm 17,4$ & $17,3 \pm 14,8$ & $22,5 \pm 17,1$ & $23,8 \pm 19,9$ & $22,3 \pm 16,5$ \\
\hline Comentário & $292,6 \pm 393,3$ & $749,4 \pm 933,6$ & $150,4 \pm 152,8$ & $324,5 \pm 285,1$ & $438,3 \pm 528,7$ & $445,2 \pm 620,4$ \\
\hline \multicolumn{7}{|l|}{ Facebook } \\
\hline Postagem & $19,1 \pm 12,1$ & $23,7 \pm 14,6$ & $21,0 \pm 12,4$ & $44,3 \pm 26,8$ & $58,8 \pm 34,0$ & $34,6 \pm 26,3$ \\
\hline Comentário & $222,5 \pm 330,5$ & $84,7 \pm 85,4$ & $111,7 \pm 148,8$ & $462,2 \pm 356,2$ & $1760,5 \pm 3370,3$ & $502,6 \pm 1498,3$ \\
\hline
\end{tabular}




\section{Total}

\begin{tabular}{lcccccc} 
Postagem & $34,6 \pm 13,3$ & $48,6 \pm 32,0$ & $34,8 \pm 25,7$ & $64,5 \pm 41,3$ & $76,1 \pm 49,1$ & $34,6 \pm 38,1$ \\
Comentário & $483,3 \pm 472,1$ & $829,6 \pm 998,6$ & $232,0 \pm 277,3$ & $762,4 \pm 641,4$ & $2079,2 \pm 3768,3$ & $891,2 \pm 1706,8$ \\
\hline
\end{tabular}

Quanto à data das postagens, destacou-se quando ocorreu a primeira publicação relacionada à COVID-19 de cada universidade, a fim de verificar quando se iniciou este debate nas suas redes sociais; além de quando a publicização da suspensão das atividades da instituição foi divulgada (Figura 1). Ressalta-se que as datas destes eventos correspondem ao que foi publicado nas redes sociais das universidades públicas federais, o que pode não representar o início real destes processos.

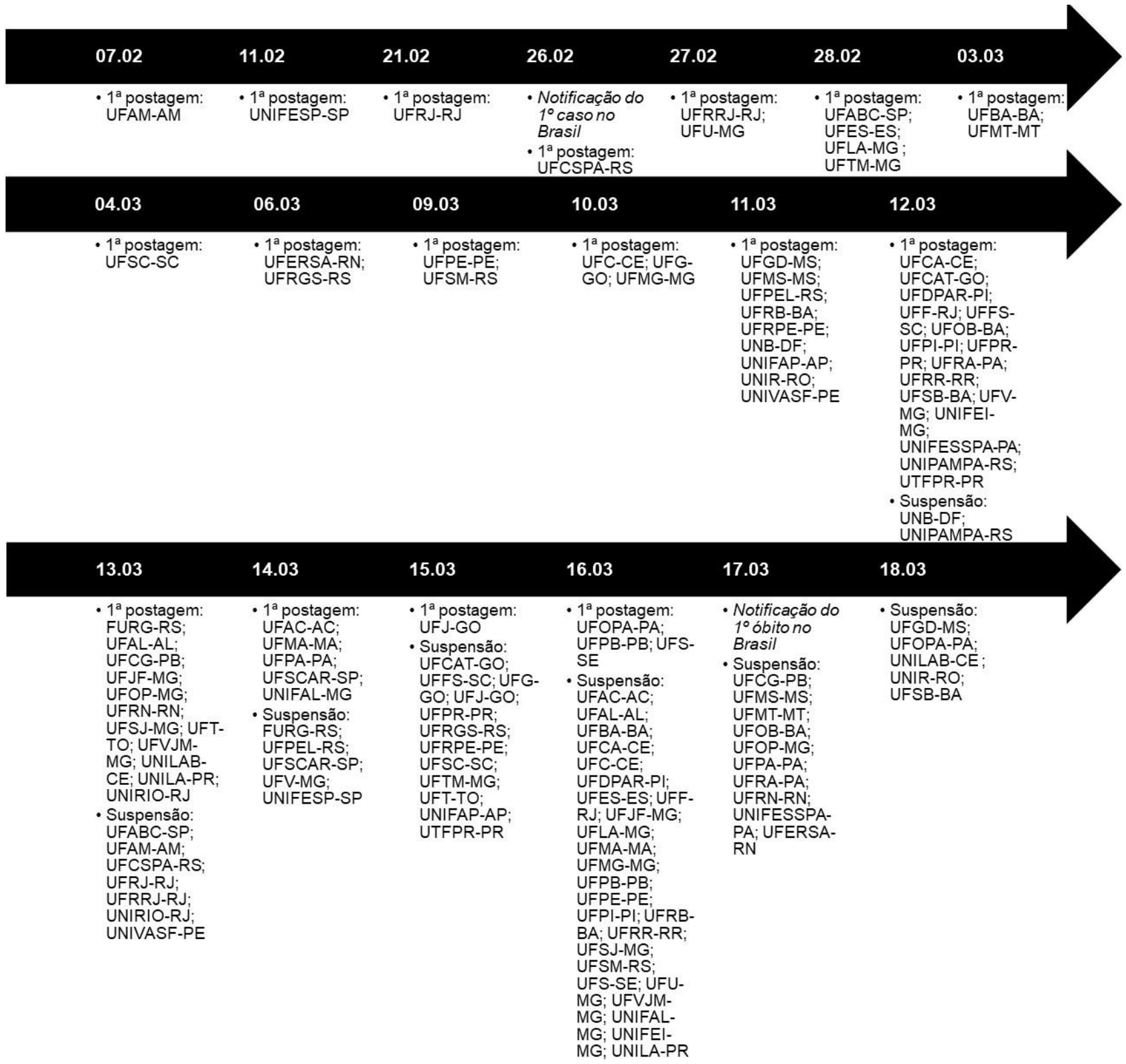

Figura 1: Linha do tempo da 1ạ publicação relacionada à COVID-19 e da divulgação da suspensão das atividades.

Verifica-se que apenas $10(15,2 \%)$ universidades divulgaram conteúdo relacionado à COVID-19 em suas redes sociais no mês de fevereiro de 2020. As que realizaram tal ação publicizaram, sobretudo, recomendações de prevenção, com divulgação de materiais do Ministério da Saúde brasileiro (UFRRJ, UFABC, UFES, UFLA, UFTM). As demais divulgaram o preparo 
da universidade para o enfrentamento à pandemia, com realização de palestras e aulas abertas (UFAM, UNIFESP, UFRJ, UFSCPA), além do envolvimento de pesquisadores da instituição em projetos relacionados ao tema (UFU).

A maioria das universidades públicas federais, porém, iniciaram a publicização de postagens relacionadas à COVID-19 apenas em março, sobretudo a partir do dia 12 de março, data em que ocorreu as primeiras divulgações de suspensão das atividades, as quais aconteceram primeiramente na UNB e na UNIPAMPA. Após isso, entre os dias 13 e 18 de março, todas as demais universidades públicas federais publicizaram a suspensão de suas atividades, inicialmente acadêmicas, com paulatina divulgação de adoção do trabalho remoto, suspensão de atendimentos presenciais e manutenção apenas dos serviços essenciais.

No que se refere ao conteúdo das postagens, estes foram classificados em ações, divulgações e recomendações. Predominou a publicização de ações realizadas pelas universidades para enfrentamento à COVID-19 (Figura 2). As divulgações envolveram: acesso gratuito a materiais de pesquisa, de formação e culturais de diversas instituições; boletins epidemiológicos dos estados; fluxo de atendimento nos estados; e materiais do Ministério da Saúde (MS) e Organização Mundial da Saúde (OMS).

\begin{tabular}{|l|}
\hline \multicolumn{1}{|c|}{ A } \\
\hline - Inscrição para estágio \\
voluntário; Página com \\
perguntas e respostas \\
sobre a COVID; \\
Realização de pesquisa \\
para verificar o bem- \\
estar da comunidade \\
acadêmica; Serviço de \\
acolhimento via telefone \\
para estudantes \\
\hline
\end{tabular}
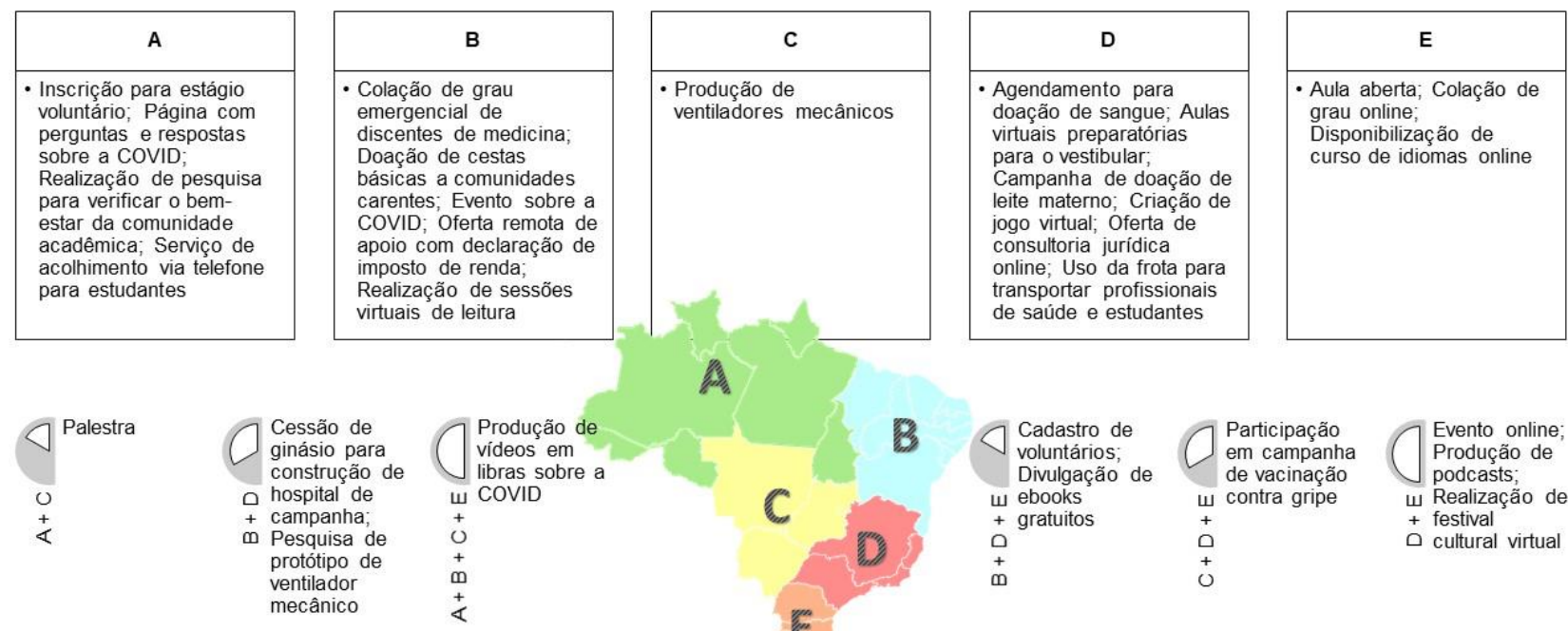

\section{$A+B+C+D+E$}

- Adoção do trabalho remoto; Apoio psicológico à comunidade acadêmica; Atuação do hospital universitário; Atuação dos docentes e discentes em ações de formação/educação e pesquisa; Atuação no diagnóstico laboratorial; Criação de grupo de enfrentamento; Criaçẩo de site com informaçōes sobre CoVID; Divulgação de canais de comunicação com a universidade; Disponibilização de cursos gratuitos online; Disponibilização de serviço de Teleatendimento; Edital para auxílio emergencial aos estudantes; Produção de álcool; Produção de Equipamento de Proteção Individual; Produção de material educativo; Realização de campanha de doação; Realização de lives com conteúdo educativo; Realização de programas na rádio e TV universitária sobre a COVID; Suspensão das atividades

Figura 2: Ações de enfrentamento à COVID-19 publicizadas nas redes sociais das universidades públicas federais brasileiras, segundo região.

As postagens com recomendações, por sua vez, englobaram orientações sobre: alimentação saudável; combate às Fake News; cuidados de higienização ao sair e ao chegar em casa; etiqueta respiratória; evitar aglomerações; evitar contato físico; ficar em casa; lavar as mãos; manter ambientes ventilados; manter distância de segurança; manter-se hidratado; não compartilhar objetos pessoais; não tocar olhos, nariz e boca; prática de exercícios físicos em casa; preservar a saúde mental; uso do álcool em gel; e uso de máscaras. Vale ressaltar que, ao longo do 
tempo, novas orientações foram adicionadas, em consonância com a dinamicidade deste processo, como foi o caso da recomendação do uso de máscaras caseiras.

No que se refere à produção de materiais educativos pelas universidades, estes envolveram guias, cartilhas, infográficos, glossários, vídeos e protocolos, englobando os temas: alimentação em época de pandemia; saúde mental na quarentena; combate às Fake News; como fabricar máscaras caseiras; como minimizar o estresse; mitos acerca da COVID; importância do investimento na ciência; aleitamento materno em tempos de COVID; uso de Equipamento de Proteção Individual; orientações para pais; como minimizar a ansiedade; guia para famílias de crianças com autismo; prática de exercícios físicos em casa; prevenção para pessoa com deficiência; como usar o álcool em gel; gravidez e a COVID; populações vulneráveis; COVID em animais; prevenção à violência intrafamiliar; orientações para cardiopatas; automedicação; ergonomia em home office; orientações para isolamento domiciliar; orientações para ida ao supermercado; como higienizar o celular; e orientações para higienizar compras.

Para analisar a repercussão da publicização das ações realizadas e divulgadas nas redes sociais das universidades públicas federais brasileiras frente à COVID-19, os comentários disponíveis foram submetidos à análise lexicográfica. O corpus textual foi composto por 26.600 textos, ou seja, comentários disponíveis nas redes sociais. Destes, 25.420 textos foram analisados pela CHD, o que denota um aproveitamento de $95,56 \%$ dos textos. O corpus textual foi repartido em duas classes, que denotaram uma mudança na repercussão das publicações após a decisão acerca da suspensão das atividades da universidade e início da publicização das ações desenvolvidas para contribuir com o enfrentamento à COVID (Figura 3).

A Classe 1, denominada "Angústias antes da suspensão das atividades e reconfigurações do funcionamento da universidade", integrou $69,97 \%$ do corpus textual, o que revela um significativo quantitativo de comentários nas publicações anteriores à suspensão das atividades e que publicizavam decisões administrativas de funcionamento da instituição após esta decisão. As palavras "aula", "saber", "esperar", "suspender", "cancelar" e "risco" foram significativas na composição da classe 1 (Figura 3A).

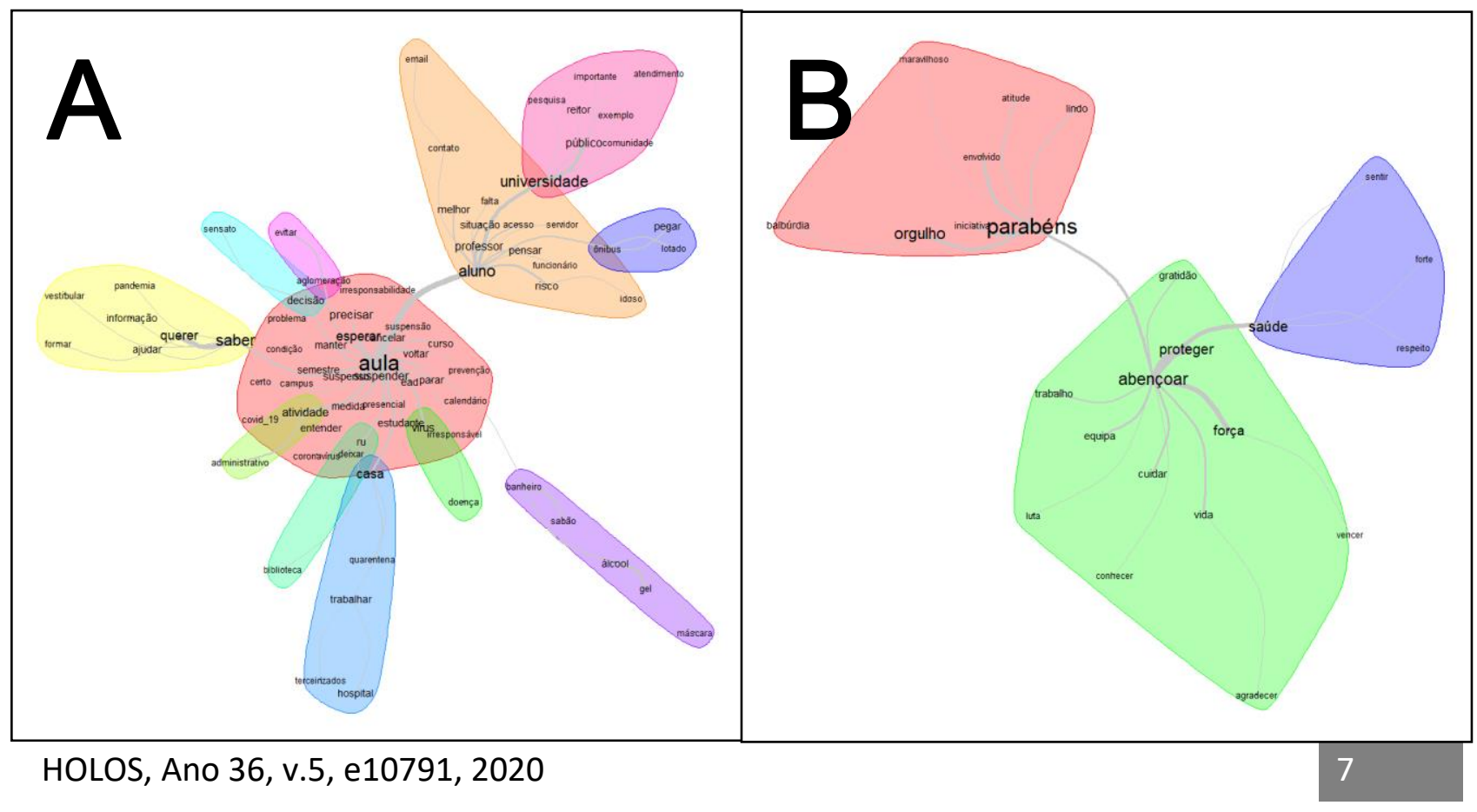


Figura 3: Análise de similitude da classe 1 (A) e da classe 2 (B).

Denota-se claramente uma repercussão de angústia e dúvidas nos comentários antes e momentos imediatos após a suspensão das aulas. Quanto aos inúmeros comentários antes da divulgação da decisão de suspensão das atividades pelas universidades, revela-se uma preocupação com a propagação do vírus, em momentos em que a transmissão comunitária já havia sido relatada em muitos estados brasileiros. Na Figura 3A, observa-se as palavras "evitar", "aglomeração" e "quarentena" que evidenciam essa preocupação, também visualizada nos comentários destacados: Decisão inadequada, outras IFES optaram pela decisão de suspender suas atividades, trata de uma situação emergencial na qual a demora na tomada de decisões de segurança pode trazer danos irreparáveis (Postagem no 3, Facebook, UFJF); Não é suspender as aulas, é suspender qualquer atividade acadêmica ou não, estudantes funcionários e trabalhadores do ambiente estão expostos (Postagem no 4, Instagram, UFCA).

Outro motivo de preocupação bastante evidenciado na classe 1 relaciona-se às carências estruturais das universidades, o que pode ser compreendido a partir do destaque dos vocábulos "banheiro, "sabão e "álcool", conforme destacado nos comentários: Nos banheiros não tem nem sabonete para lavar as mãos e vocês me vem com orientações, me poupem (Postagem no 1 , Facebook, UNB); Eu tenho uma pergunta: depois que passar a pandemia os banheiros vão voltar a ficar sem papel e sem sabão porque nunca tem (Postagem no 3, Instagram, UFMA).

No que se refere à repercussão das publicações após a decisão de suspensão das atividades das universidades, o aspecto de maior discussão e evidência foi a não adoção do Ensino a Distância (EAD) pelas instituições. Apesar de algumas falas defenderem o uso desta modalidade, predominou o elogio a não adoção, com preocupações quanto ao acesso dos estudantes, o que fica evidente nos comentários destacados: EAD sem a faculdade funcionando excluí a galera que não tem acesso à internet em casa (Postagem no 36, Facebook, UFPR); Parabéns, orgulho dessa universidade, primeiro o cuidado com a vida e não podemos nos esquecer que não é simples e rápido mudar do presencial para a EAD e, além disso, que muitos estudantes sequer têm um computador e internet em casa (Postagem no 13, Facebook, UFMG).

A Classe 2, nomeada "Orgulho pela atuação da universidade", agrupou 30,03\% dos segmentos de texto, com destaque para as palavras "parabéns", "orgulho", "balbúrdia", "gratidão" e "iniciativa" (Figura 3B). Todos os segmentos de texto incluídos nesta classe se referem às postagens acerca da publicização das ações das universidades como mecanismo de enfrentamento à COVID-19.

Os comentários incluídos na classe 2 evidenciavam aspectos positivos acerca da atuação das universidades, valorizando o papel da ciência e dos hospitais de ensino no enfrentamento à COVID-19. Traziam, ainda, críticas à desvalorização da pesquisa científica no contexto brasileiro, conforme evidenciado nos comentários: Orgulho dessa balbúrdia, todos precisando da ciência, a vida dá muitas voltas, parabéns (Postagem n 37, Instagram, UFPR); Parabéns pela iniciativa, divulgar ciência fortalece o respeito ao investimento nesse segmento (Postagem no 45, Instagram, UFU). 
Compreende-se que as redes sociais são instrumentos da contemporaneidade que propiciam interação entre os indivíduos e disseminação de informações. Frente a essas potencialidades, entidades constituintes de diversos contextos sociais, incluindo universidades, passaram a adotá-las como ferramenta de trabalho, já que essas são concebidas a partir do princípio de que o entrelaçado de ideias, interesses e recursos efetivam a comunicação, em suas diversas peculiaridades individuais, bem como gerenciam espaços virtuais a fim de construir ambientes online de encontro social (Castells, 2017; Souza, Lopes, \& Lima Filho, 2017).

Semelhantemente, observa-se que as redes sociais estão cada vez mais presentes nas estratégias comunicacionais das universidades federais brasileiras. A utilização dessas propiciam obter informações sociais, para subsidiar ações; disseminar conhecimentos, bem como as ações desenvolvidas por docentes e discentes, as atividades de retorno social e o fortalecimento da ciência.

Sobre isso, estudo brasileiro destaca que a intensidade da adoção de redes sociais por instituições de ensino aumenta a cada ano e enfatiza que os dados obtidos a partir dessas, dificilmente, seria adquirida por meio de outras modalidades de coleta de dados, já que a autodivulgação é superestimada em virtude da percepção de liberdade de comunicação pelos indivíduos (Meurer, Lopes, Antonelli, \& Colauto, 2020).

Evidenciou-se, a partir dos achados, que ocorreu um incremente de publicação a partir do dia 26 de fevereiro de 2020, o que pode estar associado ao fato da identificação do primeiro caso confirmado da COVID-19 no Brasil ter ocorrido nesse período. Paralelamente a isso, iniciam-se as pesquisas e delineamento de medidas de contenção do vírus pelos órgãos internacionais e nacionais.

Sobre essa questão, salienta-se que a OMS declarou, em 30 de janeiro de 2020, os casos da COVID-19 como uma Emergência de Saúde Pública Internacional, o que constitui o nível máximo de alerta pela organização. Em 11 de março de 2020, a doença ocasionada pelo novo coronavírus passou a ser considerada uma pandemia, situação que demandou por parte de órgãos internacionais da saúde - OPAS e OMS, por exemplo - o apoio técnico aos países (OPAS, 2020a), e estes, por sua vez, passaram a desenvolver pesquisas e outras iniciativas de produção de conhecimento.

Já as medidas de suspensão de atividades acadêmicas e administrativas e, consequentemente, implantação de trabalho remoto, ocorreu em meados do mês de março de 2020, período que coincide com a efetivação de inúmeras iniciativas, como o distanciamento social, o qual é compreendido como a melhor aposta para reduzir a transmissão e retardar a propagação do vírus em nossas comunidades (OPAS, 2020b).

Em consonância com a assertiva supracitada, estudo realizado por pesquisadores dos Estados Unidos da América e Londres destacam que o distanciamento social é adotado com fins de reduzir as interações entre pessoas na comunidade, as quais podem estar infectadas e assintomáticas. Portanto, é considerada uma medida útil nos cenários de transmissão comunitária. Todavia, trata-se de um grande desafio, já que há implicações de direitos humanos individuais (Wilder-Smith \& Freedman, 2020). 
Esses pesquisadores ressaltam a importância das mídias sociais como uma estratégia de apresentar à sociedade os motivos para a adesão ao distanciamento social, divulgação de dicas práticas de como implementá-la, bem como mitigar Fake News e o pânico na sociedade,16 enunciados que corroboram com os achados deste estudo.

Ainda, é válido destacar que o distanciamento social deve ser concatenado a medidas de suporte social aos indivíduos com maior vulnerabilidade. Com isso, é possível maximizar a adesão social a essa medida, além de assegurar os meios de subsistência (OPAS, 2020b).

Nesse sentido, para o enfrentamento à COVID-19, as universidades públicas federais brasileiras implementaram ações voltadas desde ao processo de trabalho in locus até ações direcionadas à saúde dos brasileiros. Nesse contexto, o suporte social, as práticas de fortalecimento da ciência e a atenção a questões da saúde mental são proposições pertinentes de reflexões, especialmente no contexto de uma pandemia.

Observou-se que o suporte social estava direcionado aos discentes e comunidades carentes e foi efetivado através de doação de cestas básicas, auxílio emergencial pelas universidades ao corpo discente em necessidade financeira, oferta de transporte, entre outros. Esses aspectos reafirmam que uma das missões das universidades é a resolução de problemas sociais e abertura de novas possibilidades (Audy, 2017), especialmente quando $70,2 \%$ dos discentes brasileiros recebem até um e meio salário mínimo (ANDIFES, 2020).

Ainda, no contexto do suporte social, evidenciaram-se inúmeras ações de fortalecimento do Sistema Único de Saúde (SUS), como: atuação de docentes, discentes e profissionais dos Hospitais Universitários, capacitação de recursos humanos, produção de tecnologias e insumos ventiladores mecânicos de baixo custo, produção de Equipamento de Proteção Individual (EPI), como as máscaras do tipo Face Shield, e produção de preparação alcoólica e álcool líquido à 70\% -, elaboração de fluxogramas de assistência e protocolos, concessão de equipamentos para análise laboratorial e espaços físicos para instalação de hospital de campanha para subsidiar os cuidados realizados pelos profissionais de saúde aos indivíduos vítimas da COVID-19.

Outra questão revelada quanto às ações de fortalecimento do SUS realizadas pelas universidades analisadas diz respeito à preocupação com medidas de prevenção e controle da disseminação do novo coronavírus entre profissionais de saúde. Em coerência ao exposto, a Agência Nacional de Vigilância Sanitária, com o intuito de preservar o não adoecimento desses pela COVID-19, destaca que é fundamental a disponibilização de água e sabonete líquido ou preparação alcoólica a 70\% e EPI específicos aos procedimentos, como: óculos de proteção ou protetores faciais (face shield), avental, luvas de procedimentos, gorros, N95/PFF2 ou equivalente (Brasil, 2020a).

Associado ao fortalecimento do SUS, constata-se o resgate/fortalecimento da interface entre ciência e saúde, perspectiva evidenciada nas ações educativas, por intermédio de cursos online, disponibilização de materiais educativos, vídeos em libras, entre outros; pesquisas básicas, pautadas sobretudo em estudos epidemiológicos, os quais forneceram projeções para subsidiar o planejamento das ações em saúde; e pesquisas aplicadas nos serviços, a partir do desenvolvimento de tecnologias em saúde. 
Sabe-se que a pandemia do coronavírus resulta/resultará em impactos na sociedade, já que corresponde a uma situação singular na história da humanidade. Esse cenário de insegurança desencadeia lacunas de conhecimentos, o que repercute negativamente na compreensão de cadeia epidemiológica do vírus, no tratamento e na identificação de possíveis sequelas. Posto isso, a ciência é crucial para anteparar essas fragilidades. Portanto, cientistas e pesquisadores de universidades e outras instituições deveriam ser ouvidos, valorizados e apoiados (De Negri, Zucoloto, Miranda, \& Koeller, 2020).

No tocante à saúde mental, as discussões sobre essa temática ocorreram através de múltiplas estratégias, a saber: lives, material educativo, vídeos, recursos em libras, dicas literárias, ênfase na importância da adoção de rotinas, festival cultural virtual, teleatendimento psicológico, entre outros. Essas ações de apoio são fundamentais, especialmente aquelas direcionadas para a ativação social, pois vivenciar uma pandemia gera perturbações psicossociais no tocante à capacidade de enfrentamento da problemática por parte dos indivíduos (Brasil, 2020b).

Outro aspecto a ser discutido refere-se à dinamicidade das postagens com a recomendação do uso de máscaras pela população, que, inicialmente, era restrita aos indivíduos com sinais/sintomas respiratórios, profissionais da saúde e cuidadores, não sendo aconselhado o uso em indivíduos saudáreis (Sociedade Brasileira de Pneumologia e Tisiologia, 2020).

Contudo, estudo realizado pela OMS revelou que a adesão do uso de máscaras caseiras por toda população é uma medida importante, pois evidências científicas atuais indicam que a transmissão ocorre, também, por indivíduos assintomáticos. Assim, mesmo não proporcionando proteção total contra infecções, o uso delas pode diminuir a incidência da doença, mas, para tanto, é fundamental a combinação com outras medidas preventivas - como a higienização das mãos e etiqueta respiratória (Brasil, 2020c; Oliveira, Lucas, \& Iquiapaza, 2020).

No que se refere à repercussão dos temas abordados, foi possível evidenciar que a angústia foi um elemento presente nos comentários das primeiras postagens das instituições de ensino. Isso pode ter ocorrido em virtude de que as informações, até então, eram dispersas sobre a relação dos casos confirmados no Brasil, bem como acerca da gravidade e forma de disseminação do vírus.

Além disso, as universidades estavam na fase de organização de comitês locais de acompanhamento para gerir a crise do coronavírus nas regiões correspondentes. Após a decisão de suspensão das aulas presenciais, com a finalidade principal de evitar aglomeração, de forma uníssona refletiu nos comentários de que essa foi uma atitude sensata. A suspensão das aulas presenciais não ocorreu somente no Brasil, foi uma das atitudes que grande parte das escolas e universidades do mundo optaram por fazer. De acordo com a Organização das Nações Unidas para a Educação, a Ciência e a Cultura (UNESCO, 2020), mais de 1,5 bilhão de estudantes estão sendo afetados pela pandemia em todo o mundo e, atualmente, a educação enfrenta uma crise sem precedentes. Esse panorama tem afetado alunos de todos os níveis de ensino, seja da educação básica como do nível superior.

Com isso, algumas universidades criaram um plano de contingência que busca novas formas de implementar outras modalidades de ensino, a fim de oportunizar a aprendizagem inclusiva e equitativa (Portugal, 2020). Entretanto, o Brasil enfrenta, ainda, sérios problemas relacionados a infraestrutura de forma geral, o que vem a ser um dilema perante a pandemia instalada: como 
oferecer a continuidade do ensino sem possuir estrutura física e de teletrabalho adequada para isso? Esse fato também foi observado nos resultados do estudo em tela na forma de anseio dos alunos por melhores condições físicas do ambiente universitário, como os comentários acerca de sabão e água nos banheiros das universidades.

Sabe-se que a estrutura física do ambiente acadêmico pode facilitar ou dificultar o engajamento do aluno em seu processo ensino-aprendizagem. É o que reflete estudos realizados em instituições públicas de ensino do Rio Grande do Sul, em que evidenciou que as condições físicas geram obstáculos para o bom andamento de muitas atividades acadêmicas (Pivetta et al., 2019). Além disso, essa defasagem física se deve à expansão da oferta de cursos superiores não acompanhada de investimentos adequados, o que acarretou uma infraestrutura não condizente com o atual número de alunos, bem como o fato das instalações serem muito antigas e não preparadas para a grande demanda acadêmica.

Outro aspecto mencionado nos resultados é a não adoção do EAD por parte das instituições federais públicas brasileiras. A não adesão dessa prática também é um reflexo da carência de uma estrutura inadequada para fornecer suporte a esta demanda (Arruda \& Arruda, 2015). É importante denotar que a modalidade EAD pressupõe o uso de computadores com acesso à internet banda larga e de qualidade, o que não é uma realidade para grande parte dos discentes das universidades públicas em todo o país. Existe uma desigualdade digital que não garante o acesso igualitário (Martins \& Mill, 2016). Trata-se de importante discussão no cenário atual, em que se vislumbram iniciativas e tentativas de retorno gradual das atividades acadêmicas das universidades, a partir de estratégias de suporte aos discentes e docentes numa perspectiva de ensino remoto.

Outro ponto evidenciado no presente estudo foi o orgulho pela atuação das universidades perante o enfrentamento da pandemia. É importante denotar que a condução e manutenção da pesquisa brasileira vem passando por um momento difícil na atualidade. O enfrentamento se dá pela desvalorização da ciência, cortes e contingenciamento de verbas para se fazer estudos científicos no ambiente universitário.

Esse presente cenário acabou por evidenciar uma crise aguda não só de origem patológica, mas também de falta de confiança nos seres humanos (Harasim, 2005). Isso acontece por carência de líderes que minaram a confiança na ciência, nas instituições e na cooperação internacional, o que pode ser reportado nos comentários de forma irônica o termo "balbúrdia". É importante destacar que, perante essa crise, a universidade ganhou um novo impulso de mostrar que também está na linha de frente, realizando pesquisa de ponta mesmo com parcos recursos disponíveis.

Além disso, passou a estimular a disseminação do conhecimento com a proposição de seminários de acesso aberto e gratuito, formação de construção de parcerias internacionais, alteração da rotina e estabelecendo prioridades para dar continuidade as ações mesmo em meio a pandemia. $O$ que faz refletir que a proteção real vem da cooperação global e da confiança na pesquisa e que a produção do conhecimento está hoje associada à construção coletiva em prol da pesquisa científica de qualidade (De Negri et al., 2020). Por isso, o orgulho e a satisfação em fazer parte de um momento histórico em que até mesmo o ambiente acadêmico teve que se renovar e revigorar. 
Quanto às limitações desta pesquisa, destacam-se: os dados representam apenas o que foi publicizado nas redes sociais das universidades e, portanto, não concebem a totalidade das ações e contribuições destas instituições; em relação aos comentários das postagens, verificou-se que o número disponível não condizia com o quantitativo sinalizado em ambas redes sociais; é importante compreender que as repercussões discutidas refletem apenas a concepção dos usuários das redes sociais que possuem o hábito de comentar em postagens; foram analisados as postagens apenas até o dia 06 de abril, o que representa, em média, os 20 primeiros dias de atuação das universidades após a suspensão de suas atividades, destarte, os resultados apresentados devem ser compreendidos nesta perspectiva, pois muitas novas ações já foram consolidadas.

\section{CONCLUSÃO}

Elucidou-se que as universidades públicas federais brasileiras têm atuado ativamente em ações de enfrentamento à COVID-19, desde reorganizações acadêmicas e administrativas para contribuir com a minimização do risco a sua comunidade e região, até ações sociais de suporte à comunidade acadêmica, sobretudo estudantes, bem como pesquisas básicas e aplicadas relacionadas à temática.

Quanto às repercussões destas ações, evidenciou-se um momento de angústia da comunidade universitária antes da suspensão das atividades das instituições, o que foi seguido por dúvidas e anseios quanto à reorganização acadêmica e administrativa. Todavia, ganhou destaque nos comentários o imenso orgulho da atuação das universidades no enfrentamento à COVID-19, com uma importante reflexão acerca do papel da ciência neste panorama e do necessário investimento nesta área.

\section{REFERÊNCIAS}

Arruda, E. P. \& Arruda, D. E. P. (2015). Educação à distância no Brasil: políticas públicas e democratização do acesso ao ensino superior. Educação em Revista, 31(3), 321-338. doi:10.1590/0102-4698117010

Associação Nacional dos Dirigentes das Instituições Federais de Ensino Superior. (2020). V pesquisa de perfil socioeconômico e cultural dos estudantes de graduação das instituições federais de ensino superior brasileiras. Recuperado de: http://www.andifes.org.br/wpcontent/uploads/2019/05/V-Pesquisa-do-Perfil-Socioecon\%C3\%B4mico-dos-Estudantes-deGradua\%C3\%A7\%C3\%A3o-das-Universidades-Federais-1.pdf

Audy, J. (2017). A inovação, o desenvolvimento e o papel da Universidade. Estudos Avançados, 31(90), 75-87. doi:10.1590/s0103-40142017.3190005

Barcelos, P. E. L., Lima, T. V., \& Aguiar, A. C. (2020). Blogs e redes sociais na atenção à saúde da família: o que a comunicação online traz de novo? Rev Eletron Comun Inf Inov Saúde, 14(1), 126-149. doi:10.29397/reciis.v14i1.1747 
Barreto, M. L., Barros, A. J. D., Carvalho, M. S., Codeço, C. T., Hallal, P. R. C., Medronho, R. A., Struchiner, C. J., Victora, C. G., \& Werneck, G. L. (2020). O que é urgente e necessário para subsidiar as políticas de enfrentamento da pandemia de COVID-19 no Brasil? Rev Bras Epidemiol. 23, e200032. doi:10.1590/1980-549720200032

Brasil. (2020a) Ministério da Saúde. Agência Nacional de Vigilância Sanitária. Nota técnica GVIMS/GGTES/ANVISA no 04/2020 orientações para serviços de saúde: medidas de prevenção e controle que devem ser adotadas durante a assistência aos casos suspeitos ou confirmados de infeç̧ão pelo novo coronavírus (SARS-CoV-2). Recuperado de http://portal.anvisa.gov.br/documents/33852/271858/Nota+T\%C3\%A9cnica+n+042020+GVIMS-GGTES-ANVISA-ATUALIZADA/ab598660-3de4-4f14-8e6f-b9341c196b28

Brasil. (2020b). Ministério da Saúde. Saúde Mental e Atenção Psicossocial na Pandemia COVID-10: recomendações para gestores. Recuperado de https://www.arca.fiocruz.br/bitstream/icict/41030/2/Sa\%c3\%bade-Mental-eAten\%c3\%a7\%c3\%a3o-Psicossocial-na-Pandemia-Covid-19-recomenda\%c3\%a7\%c3\%b5espara-gestores.pdf

Brasil. (2020c). Ministério da Saúde. Agência Nacional de Vigilância Sanitária. Orientações gerais Máscaras faciais de uso não profissional. Recuperado de http://portal.anvisa.gov.br/documents/219201/4340788/NT+M\%C3\%A1scaras.pdf/bf43018 4-8550-42cb-a975-1d5e1c5a10f7

Camargo, B. V. \& Justo, A. M. (2018). Tutorial para uso do software Interface de R pour les Analyses Multidimensionnelles de Textes et de Questionnaires. Recuperado de http://iramuteq.org/documentation/fichiers/tutoriel-portugais-22-11-2018

Castells, M. (2017). Redes de indignação e esperança: movimentos sociais na era da internet. Recuperado de https://zahar.com.br/sites/default/files/arquivos/teaser_CASTELLS_RedesDelndignacaoEEsp eranca.pdf

De Negri, F., Zucoloto, G., Miranda, P., \& Koeller, P. (2020). Ciência e Tecnologia frente à pandemia: Como a pesquisa científica e a inovação estão ajudando a combater o novo coronavírus no Brasil e no mundo. Recuperado de http://www.ipea.gov.br/cts/pt/central-deconteudo/artigos/artigos/182-corona

Feitosa, L. R. C. \& Araújo, C. M. M. (2015) Qualitative research in a Web-based contextVirtual Ethnography in debate. Atas CIAQ, 3, 384-385.

Freitas, A. R. R., Napimoga, M., \& Donalisio, M. R. (2020). Assessing the severity of COVID-19. Epidemio. Serv Saúde, 29(2), e2020119. doi:10.5123/s1679-497420200002000

Gallasch, C. H., Cunha, M. L., Pereira, L. A. S., \& Silva-Junior, J. S. (2020). Prevention related to the occupational exposure of health professionals workers in the COVID-19 scenario. Rev enferm UERJ, 28, e49596. doi:10.12957/reuerj.2020.49596

Gimenez, A. M. N., Sousa, G., \& Feltri, R. B. (2020). Universidades Brasileiras e Covid-19: fortalecendo os laços com a sociedade. Recuperado de 
https://www.unicamp.br/unicamp/coronavirus/universidades-brasileiras-e-covid-19fortalecendo-os-lacos-com-sociedade

Harasim, L. (2005). Redes de Aprendizagem. São Paulo: SENAC.

Martins, S. L. B. \& Mill, D. (2016). Estudos científicos brasileiros sobre a educação a distância no Brasil: um breve panorama. Inc Soc, 10(1), 119-131.

Meurer, A. M., Lopes, I. F., Antonelli, R. A., \& Colauto, R. D. (2020). Experiences in Graduate Studies, Behavior in Social Networks and Well-Being. Educ Realidade, 45(1), e86158. doi:10.1590/2175-623686158

Oliveira, A. C., Lucas, T. C., \& Iquiapaza, R. A. (2020). O que a pandemia da covid-19 tem nos ensinado sobre adoção de medidas de precaução? Texto Contexto Enferm, 29, e20200106. doi:10.1590/1980-265x-tce-2020-0106

Organização das Nações Unidas para a Educação, a Ciência e a Cultura. (2020). No Dia da Educação, UNESCO chama atenção para urgência de enfrentar desafios impostos pela COVID-19. Recuperado de https://nacoesunidas.org/no-dia-da-educacao-unesco-chama-atencao-paraurgencia-de-enfrentar-desafios-impostos-pela-covid-19/amp/

Organização Pan-Americana de Saúde. (2020a) Folha informativa - COVID-19 (doença causada pelo novo coronavírus). Recuperado de https://www.paho.org/bra/index.php?option=com_content\&view=article\&id=6101:covid19 \&ltemid $=875$

Organização Pan-Americana de Saúde. (2020b). COVID-19: Diretora da OPAS pede 'extrema cautela' na transição para medidas de distanciamento social mais flexíveis. Recuperado de https://www.paho.org/bra/index.php?option=com_content\&view=article\&id=6145:covid19-diretora-da-opas-pede-extrema-cautela-na-transicao-para-medidas-de-distanciamentosocial-mais-flexiveis\&ltemid=812

Pivetta, H. M. F., Schlemmer, N., Roveda, P. O., Isaia, S. M. A., Porolnik, S., \& Cocco, V. M. (2019). Setbacks of University Teaching in Health Sciences. Educ Realidade, 44(1), e75639. doi:10.1590/2175-623675639

Portugal. (2020). Gabinete no ministro da ciência, tecnologia e ensino superior. Recomendação e esclarecimento às instituições científicas e de ensino superior: Elaboração de planos para levantamento progressivo das medidas de contenção motivadas pela pandemia COVID-19. Recuperado de https://www.uminho.pt/PT/viver/COVID-19/Paginas/default.aspx

Rafael, R. M. R., Neto, M., Carvalho, M. M. B., David, H. M. S. L., Acioli, S., \& Faria, M. G. A. (2020). Epidemiology, public policies and Covid-19 pandemics in Brazil: what can we expect? Rev Enferm UERJ, 28, e49596. doi:10.12957/reuerj.2020.49570

Santos, V. L. C., Santos, J. E. (2014). As redes sociais digitais e sus influência na Sociedade e Educação contemporâneas. Rev Holos, 6(30), 307-328. doi:10.15628/holos.2014.1936 
Silva, A. A. M. (2020). On the possibility of interrupting the coronavirus (COVID-19) epidemic based on the best available scientific evidence. Rev Bras Epidemiol, 23, e200021. doi:10.1590/1980549720200021

Sociedade Brasileira de Pneumologia e Tisiologia. (2020). Orientações da OMS para prevenção da COVID-19. Recuperado de https://sbpt.org.br/portal/covid-19-oms/

Souza, F. B., Lopes, M. G. Q., \& Lima Filho, R. M. (2017). Redes sociales en el aprendizaje de la odontología: opinión de los estudiantes de una universidad brasileña. Rev Cubana Estomatol, 54(2), 1-11.

Wilder-Smith, A. \& Freedman, D. O. (2020). Solation, quarantine, social distancing and community containment: pivotal role for old-style public health measures in the novel coronavirus (2019nCoV) outbreak. J Travel Med, 27(2), 1-4. doi:10.1093/jtm/taaa020

\section{COMO CITAR ESTE ARTIGO:}

Salvador, P. T. C. de O., Alves, K. Y. A., Rodrigues, C. C. F. M., Oliveira, L. V. e, Sousa, V. R. M.de. (2020). Ações e repercussões das primeiras iniciativas das universidades públicas federais brasileiras frente à COVID-19. Holos. 36 (6), 1-17.

\section{SOBRE OS AUTORES}

\section{P. T. C. DE O. SALVADOR}

Enfermeira, Doutora em Enfermagem, Docente da Escola de Saúde da UFRN. E-mail: petalatuani@hotmail.com

ORCID ID: https://orcid.org/0000-0002-3208-6270

\section{K. Y. A. ALVES}

Enfermeira, Doutora em Enfermagem, Docente da Escola de Saúde da UFRN. E-mail: kisnayasmin@hotmail.com

ORCID ID: https://orcid.org/0000-0001-7900-0262

\section{C. F. M. RODRIGUES}

Enfermeira, Doutora em Enfermagem, Docente da Escola de Saúde da UFRN. E-mail: claudiacrisfm@yahoo.com.br

ORCID ID: https://orcid.org/0000-0001-5182-2491

\section{V. E OLIVEIRA}

Enfermeira, Doutora em Saúde Coletiva, Docente da Escola de Saúde da UFRN. E-mail: lannuzyacg@hotmail.com

ORCID ID: https://orcid.org/0000-0002-6881-898X

\section{R. M.DE SOUSA}

Bióloga. Graduanda em Gestão Hospitalar - UFRN. Mestre em Administração pela UFRN. E-mail: soumaciel@gmail.com

ORCID ID: https://orcid.org/0000-0003-2680-0009

Editor(a) Responsável: Francinaide de Lima Silva Nascimento

Pareceristas Ad Hoc: LENINA SILVA E EDIVAL TEIXEIRA 


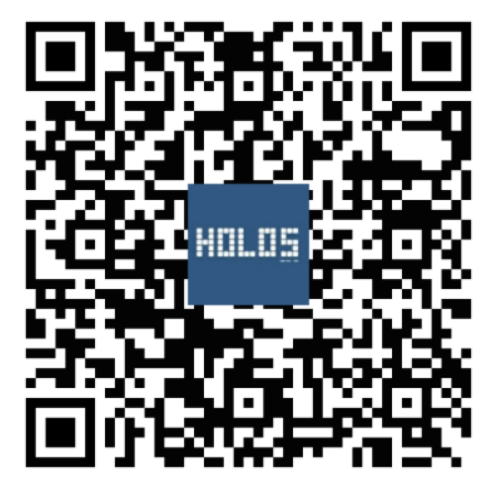

